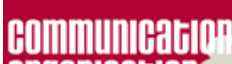

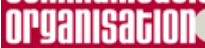

\title{
Communication et organisation
}

Revue scientifique francophone en Communication

organisationnelle

$50 \mid 2016$

Engagement entrepreneurial et territoires

\section{Pratiques et territoires de l'écoresponsabilité : des questions de gouvernance, de culture, de société et de recherche}

Le cas de la Méditerranée : matérialiser, symboliser, agir

Eco-responsibility practices and territories: questions of governance, culture, society and research. The case of the Mediterranean: materialize, symbolize, act

\section{Françoise Bernard}

\section{OpenEdition}

Journals

Édition électronique

URL : http://journals.openedition.org/communicationorganisation/5340

DOI : 10.4000/communicationorganisation.5340

ISBN : 979-10-300-0127-3

ISSN : 1775-3546

Éditeur

Presses universitaires de Bordeaux

Édition imprimée

Date de publication : 1 décembre 2016

Pagination : 45-60

ISBN : 979-10-300-0069-6

ISSN : 1168-5549

Référence électronique

Françoise Bernard, «Pratiques et territoires de l'écoresponsabilité : des questions de gouvernance, de culture, de société et de recherche », Communication et organisation [En ligne], 50 | 2016, mis en ligne le 01 décembre 2019, consulté le 16 février 2021. URL : http://journals.openedition.org/

communicationorganisation/5340; DOI : https://doi.org/10.4000/communicationorganisation.5340 


\section{Pratiques et territoires de l'écoresponsabilité: des questions de gouvernance, de culture, de sociēté et de recherche}

Le cas de la Méditerranée: matérialiser, symboliser, agir

\section{Fronçoise Bernord ${ }^{1}$}

«La Méditerranée est la mer de la communication et du conflit»

(Morin, 1998-99, 33)

La Méditerranée, «la mer aux trois rives:l'africaine, l'asiatique,l'européenne» (Morin 1999, 33) est appréhendée par les populations qui occupent ses littoraux, comme un espace naturel, économique, culturel mais aussi comme un espace de conflits et de paix et cela depuis des siècles. Le propos semble banal, mais il revêt cependant une très forte actualité avec la prise en compte des phénomènes ${ }^{2}$ associés aux mouvements migratoires ${ }^{3}$, aux crises politiques et économiques, aux questions environnementales. Cette actualité a pour effet de montrer une fois de plus que ce qui se passe au loin a des impacts très proches et inversement.

Nous nous concentrerons plutôt sur les questions environnementales particulièrement vives qui ont, dans notre réflexion, un statut d'analyseur de questions fortes de communication.

La Méditerranée est volontiers définie comme mosaïque de territoires et de cultures entre unité et diversité, définition explorée avec brio par les historiens, les travaux qui lui ont été consacrés sont devenus emblématiques de la prestigieuse École des Annales. Celle-ci mettait en œuvre un renouveau

1 Françoise Bernard, Professeure de Sciences de l'information et de la communication (SIC) à Aix-Marseille Université, dirige l'IRSIC (EA 4262). Spécialisée en communication des organisations et des institutions, elle a conduit et publié de nombreux travaux portant notamment sur les problématiques du changement et sur la communication engageante. Depuis quelques années, elle apporte également une contribution à l'étude des questions communicationnelles liées aux pratiques environnementales (changements de valeurs, de comportements...). Impliquée dans des responsabilités nationales, elle a présidé la SFSIC ainsi que la section 71 du CNU.

2 Pour une projection dans le XXI' siècle de la Méditerranée : Fabre (2014).

3 Cf. Baby-Collin V., Bensaâd A. \& Sintès P. (2009). 
de la méthode historique et proposait une réorientation qualifiée d'histoire sociale marquant un rapprochement entre l'histoire et les autres sciences sociales et humaines. Un nom est évidemment plus particulièrement attaché à ce chantier de recherche, celui de Fernand Braudel qui avait fait de la Méditerranée le sujet de sa thèse soutenue en 1947 et intitulée: "La Méditerranée et le monde méditerranéen à l'époque de Philippe II ». Le grand historien avait proposé de nouvelles perspectives et notions, parmi lesquelles nous retenons la notion de "civilisation matérielle " qui met en lumière les interdépendances entre conditions matérielles de vie -conditions naturelles et techniques prises ensemble - et phénomènes de civilisation. Dans la continuité de la pensée braudélienne, il nous semble intéressant d'ajouter à l'approche interdisciplinaire les enjeux environnementaux et de les interpréter comme des enjeux de société, de culture et de communication relevant des contradictions d'un "vivre ensemble " territorialisé dans une forme globale qui peut être qualifiée de chaotique ${ }^{4}$ et qui est traversée par les questions du temps long.

Dans le cadre d'une thématique environnementale qui, plus que toute autre, appelle une grille d'intelligibilité multidimensionnelle, où méta, macro, méso et microanalyses se croisent et s'entrecroisent, nous organisons une réflexion autour d'un objet " inter-territoires ", le déchet industriel en Méditerranée avec le cas des boues rouges examiné en Corse puis en Provence. Le déchet est aussi un révélateur des tensions et frontières entre territoires et cultures, dans une acception large (cultures régionales, cultures de métiers, culture d'écoresponsabilité, etc.).

Cette réflexion est issue d'une analyse de corpus médiatique (et en arrièreplan, d'une quinzaine d'années de projets financés et de publications consacrés à la question de la communication environnementale). Elle est complétée par une réflexion portant sur les conditions et modalités pour franchir des frontières entre territoires, entre organisations et entre cultures.

\section{Le déchet industriel un «objet inter-territoires»: le cas des boues rouges en Corse}

Nous proposons de définir les déchets issus de la production industrielle et de la consommation comme des «objets inter-territoires» qui circulent notamment de la terre vers la mer, de l'artefact industriel vers les organismes biologiques. Ils sont aussi des «objets frontières» qui mettent en relation plusieurs territoires (littoral et milieu marin), univers et groupes d'acteurs : les entreprises, les pouvoirs publics, les médias, la société civile, les chercheurs... Nous explorons un cas, celui des boues rouges en Méditerranée.

$4 \mathrm{Cf}$ : : la $22^{\mathrm{e}}$ édition des Rencontres d'Averroès sur le thème : "Méditerranée, un rêve brisé ?", cette édition avait d'ailleurs dû être annulée suite aux attentats de Paris en novembre 2015. http://culture.marseille.fr/actualites/ la-mediterranee-au-coeur-des-rencontres-d-averroes 
Les boues rouges en Corse avaient occupé la scène médiatique et politique en 1972, il s'agissait alors de rejets industriels provenant du littoral italien. Ce cas est intéressant car il illustre les tensions environnementales, entre certaines logiques entrepreunariales d'une part, et, d'autre part, des logiques écologiques et des logiques sociétales. Il est aussi représentatif des tensions entre secteurs d'activité, en l'occurrence, la pêche, le tourisme et l'industrie métallurgique. Il souligne également comment la question environnementale est adossée à d'autres enjeux qui peuvent être culturels, identitaires, sociopolitiques, etc.

Pour présenter ce cas, nous faisons référence à une production audiovisuelle originale issue d'archives de l'INA problématisées et thématisées par les chercheurs conduits par Françoise Albertini ${ }^{5}$ de l'Università di Corsica - Pasquale Paoli, dans le cadre d'un programme de recherche ${ }^{6}$. Cette production, composée de 80 documents de l'INA se présentant sous la forme "d'une fresque médiatique », est disponible en ligne 7 . Elle s'intitule : «Médias \& mobilisations en Corse, de 1945 à nos jours ». Les chercheurs présentent la production de la manière suivante : «Entre enjeu de luttes et vitrine de ces enjeux /.../ ce Tempo pose la question générique de la représentation médiatique $^{8}$ de la Corse sur plus d'un demi-siècle».

Les catégorisations sont à la fois diachroniques et thématiques. Dans le thème des "Émergences environnementales ", un dossier saillant, au début des années 1970, est celui des boues rouges. L'entreprise italienne Montedison, installée à Scarlino (littoral toscan), déverse dans les eaux de la Méditerranée des boues toxiques chargées d'oxyde de fer et d'acide sulfurique (résidus de l'exploitation d'un gisement de bioxyde de titane et de vanadium). Le déversement s'effectue, dans un premier temps, sous une forme solide et liquide, puis dans un deuxième temps, liquide : « entre 1500 et 3000 tonnes de déchets toxiques déversés chaque jour au large du Cap Corse ». Les pêcheurs touchés par la pollution sétaient mobilisés dans les ports corses, mais aussi à Marseille. Des comités " anti-boues rouges » s'étaient constitués, composés notamment de pêcheurs, scientifiques, médecins, juristes, la population avait manifesté son soutien. Parmi les archives mises en ligne, un film est intitulé: «Les Corses voient rouge ». "Ce film-enquête recueille les revendications unanimes des pêcheurs corses et toscans contre les activités de l'usine chimique Montedison de Scarlino qui détruisent l'équilibre marin ».

Deux colloques sont également mentionnés dans la fresque. Un document, consacré à la " Conférence mondiale à Beyrouth (1972) " pour la protection de la mer Méditerranée contre la pollution, témoigne combien les thèmes abordés cette année-là le seront de manière récurrente pendant les 45 années qui suivront. Il est notamment rappelé que les 132 grandes villes du pourtour

5 Enseignant-chercheur à l'UMR CNRS LISA 6240.

6 Programme financé à travers le CPER 2012-2014.

$7 \mathrm{http} / / \mathrm{m} 3 \mathrm{c}$.univ-corse.fr/omeka/medias-et-mobilisations-en-corse-de-1945-a-nos-jours

8 Sur le thème du traitement médiatique du « Problème corse », lire Lisa d'Orazio (2011), UMR LISA. 
méditerranéen comportant 100 millions d'habitants (et 200 millions en 2000) rejettent leurs détritus et leurs déchets en Méditerranée. Il est déjà question d'élaborer une charte qui devrait être approuvée par l'ONU. En 1973, un autre « colloque international sur les boues rouges à Bastia » ambitionne de servir «de base pour entreprendre une lutte générale contre la pollution» Les contributeurs déclarent l'utilité d'envisager: «des mesures répressives financières et pénales et préventives». Une convention internationale régionale impliquant les scientifiques était annoncée à l'issue du Colloque. Les déclarations se multipliaient, elles ressemblaient déjà étrangement à toutes celles qui seront faites sur ces thèmes par la suite :

«Faire appel de la décision mortelle contre la mer /.../ cessez de considérer la mer comme une poubelle /.../» (Bombard). La première séquence s'était terminée en 1974 par un premier jugement du tribunal de Livourne, mais l'épilogue judiciaire avait eu lieu en 1985, censé mettre un terme aux agissements de la multinationale Montedison.

L'étude du corpus audio-visuel permet d'identifier certaines caractéristiques narratives et argumentatives que nous résumons de la manière suivante:

- la crédibilité de la source: les pêcheurs des spécialistes de la mer au quotidien, aux côtés d'autres spécialistes : des scientifiques;

- le colloque comme dispositif de médiation, d'intermédiation et de médiatisation

- la preuve par le visible : des cétacés morts s'échouant sur les côtes

- le rôle de l'invisible : dévoiler ce que l'on nous cache

- le cumul des difficultés et des épreuves : crise environnementale s'ajoutant aux nombreuses autres difficultés de l'île

- l'inquiétude pour l'avenir formulée dans les termes de : «l'avenir de nos enfants "

- l'imaginaire de la mer nourricière pour les générations du passé, du présent, du futur

- l'urgence à agir

- la mobilisation élargie comme disruption : demain sera différent.

Certains de ces éléments constitutifs du tissu narratif se retrouveront dans d'autres situations où les thématiques des déchets sont présentes. Cependant, il convient de noter les spécificités culturelles liées au contexte corse et aux capacités de mobilisation de sa population. Il est intéressant de souligner, au travers de cet exemple, que le thème environnemental est repris dans le cadre d'une approche systémique : pollution marine, destruction de la faune, emploi, place de la population corse dans le « développement » économique, contributions scientifiques, etc. Au sein de ce système signifiant lié à une forme inter-organisationnelle éphémère, celle des mobilisations, émerge plus fortement la question de l'identité culturelle corse qui réorganise 
l'ensemble des éléments et produit des effets de symbolisation ${ }^{9}$. Il est également intéressant de souligner combien les questions de l'action et de la responsabilité environnementales sont adossées à la constitution de formes ${ }^{10}$ organisationnelles plurielles et ouvertes composées de "parties prenantes » diverses (Bernard, 2013).

Récemment le cas des boues rouges en Méditerranée rejetées cette fois-ci, non pas entre l'Italie et la Corse, mais dans le Parc naturel des Calanques a resurgi sur la scène médiatique et a fait l'objet d'un pic médiatique à l'automne 2015.

\section{Le «retour» des boues rouges, en Provence en 2015: quid de la responsabilité publique et entrepreunariale?}

Nous aurions pu ajouter comme sous titre: lorsque l'histoire ne se répète jamais tout à fait, car ce qui fait la différence avec le cas précédent, c'est que la pollution se produit dans un « territoire protégé et sanctuarisé ». En Provence, le thème revient en fin d'année 2015 , à l'issue d'une enquête publique ${ }^{11}$, avec l'autorisation préfectorale et donc celle du gouvernement, dans le département Bouches-du-Rhône, permettant la poursuite pendant six ans du rejet de boues rouges issues de la production d'alumine, déchets déversés en Méditerranée dans les eaux du Parc national des Calanques ${ }^{12}$. Le Ministère de l'Écologie avait anticipé et réagi en sollicitant des organismes experts pour étudier le dossier ${ }^{13}$. Les conclusions de ces organismes avaient servi à éclairer la décision gouvernementale. Cette décision officielle de l'État intervient alors que, après 50 ans de rejet de boues rouges dans la Méditerranée et conformément à la Convention internationale de Barcelone pour la protection de la Méditerranée, l'usine Alteo ${ }^{14}$ aurait dû cesser tout rejet solide en mer au 1er janvier 2016. La décision s'inscrit dans un contexte médiatique où d'autres points de vue s'expriment et notamment l'inquiétude des riverains et l'indignation des défenseurs de l'environnement. L'entreprise Alteo de son côté «déplore une

9 Cf. Batazzi C. \& Masoni Lacroix C. (2008) qui proposent d'inscrire les pratiques organisationnelles dans un ordre symbolique transcendant techniques, structures et immédiateté.

10 Sur la question des formes plus généralement, cf. Lardellier (2014).

11 « Le 22 décembre, le Conseil supérieur de prévention des risques technologiques, chargé d'une enquête publique concernant 27 communes, avait déjà rendu un avis favorable aux demandes d'Alteo ». En savoir plus sur :http://www.lemonde.fr/pollution/article/2015/12/29/le-prefet-autorise-le-rejet-des-boues-rouges-dansle-parc-national-des calanques_4839315_1652666.html\#Crufrz8sPkib8Tdh.99

12 Le Parc national des Calanques, terrestre, marin et périurbain créé en 2012 est le dixième parc national en France. Sur le site officiel, rubrique espaces presse: 58 documents sont en ligne, dont un consacré aux boues rouges (11/6/2013) ; il est précisé que : «L'espace marin concerné /par les boues rouges/ est classé par décret, depuis le 20 avril 2012, en cœur du Parc national des Calanques /.../ 23 millions de m3 de résidus déversés dans le canyon au cours des 45 dernières années ».

13 En savoir plus sur http://www.lemonde.fr/pollution/article/2014/10/20/segolene-royal-a-la-chasse-auxboues-rouges-de-gardanne_4509213_1652666.html\#CYSpOrzXbiwUMS1Y.99

14 L'usine Alteo est certifiée OHSAS 18001 (Santé et Sécurité), ISO9001 (Qualité) et ISO14001 (Environnement), source : http:/www.alteo-alumina.com/fr/gardanne ; l'entreprise a créé un site « environnement » : http://www.alteo-environnement-gardanne.fr/ 
campagne de désinformation $»^{15}$ et rappelle que: «L'arrêté prévoit en outre un suivi renforcé notamment par la création d'un Comité de suivi de site et d'une Commission de surveillance et d'information sur le rejet marin ». Les responsables de l'usine insistent sur le maintien des emplois et sur le fait que les rejets sont des effluents, de "l'eau de rinçage », que les déchets solides seront transformés « en bauxaline, un matériau que l'entreprise pourra revendre pour la fabrication de tuiles ou de béton léger ». Grâce à ce projet de « recyclage des déchets solides », l'entreprise peut se présenter comme acteur de l'économie circulaire, montrant ainsi son sens de la « responsabilité ». Enfin, le Parc national des Calanques avait donné son autorisation accompagnée de conditions : «Alteo doit désormais, tous les ans, remettre un rapport détaillé sur ses activités et a jusqu'à 2018 pour réduire, voire supprimer les polluants présents ».

La presse quotidienne régionale ${ }^{16}$ et nationale ${ }^{17}$ relaye largement cette décision en soulignant, assez souvent, les tensions internes au gouvernement sur ce dossier. L'arrêté prescrit également la poursuite de l'amélioration de la qualité des rejets liquides résiduels afin de les rendre conformes aux normes en vigueur d'ici au 31 décembre 2021 au plus tard ». Signe des temps, même si l'information se fait plus technique, nous retrouvons du côté des opposants, trente plus tard une structure narrative qui présente certains points communs avec celle des boues rouges en Corse. Mais bien sûr, il convient d'ajouter à l'orchestre médiatique "classique ", les contributions des réseaux sociaux et l'interactivité numérique ${ }^{18}$. Nous ne proposons pas une analyse de ces contenus numériques ici. Cependant, une grande différence apparaît, celle de l'argument de l'emploi industriel direct et indirect et, par exemple, on note la déclaration du PCF13, via le journal La Marseillaise, qui en appelle à « l'avancement des recherches en matière de dépollution » pour favoriser la préservation « de la filière économique de l'alumine pour maintenir l'emploi $»^{19}$. La déclaration est pour le moins révélatrice de paradoxes : accepter la pollution avec des risques d'irréversibilité pour le milieu marin (effets de cumulation et d'intensification des pollutions), pour sauver des emplois industriels à court terme sur terre. La déclaration exprime aussi un acte de foi adressé aux scientifiques et à l'ingénierie «verte » supposés résoudre «techniquement» les problèmes environnementaux.

Nous pointons par ailleurs, un effet communicationnel bien connu des chercheurs, celui de l'agenda setting (McCombs \& Shaw 1972), le thème des

15 http://www.alteo-environnement-gardanne.fr/ ; Plummer W., Querelle entre Royal et Valls sur le rejet des boues rouges dans les Calanques, Le Figaro, 30/12/2015.

16 En ce qui concerne la PQR, cf. notamment : Benallal H., Colère verte contre les « Boues rouges » d'\#Altéo, La Marseillaise, 1/2/2016 ; http://www.laprovence.com/t/boues-rouges

17 Cf. notamment : Landrevie B., La Méditerranée empoisonnée, mai 2015, Le Monde diplomatique, http:// www.monde-diplomatique.fr/2015/05/LANDREVIE/52952

18 Cf. notamment : http://www.huffingtonpost.fr/daniele-favari/boues-rouges-colere-noire_b_9154894.html 19 http://www.lamarseillaise.fr/bouches-du-rhone/developpement-durable/46366 
boues rouges sort de l'indifférence en étant saisi par la presse (PQR et PQN, les médias) à l'automne 2015. Or, ce cas avait commencé dans les années 1960 et n’avait jamais cessé depuis. Plus généralement encore, ce thème a une histoire longue en Provence. Pas loin de Gardanne, à Marseille, des historiens ont montré que la présence de l'alumine fait partie du patrimoine industriel de la Ville depuis 1893 (Mioche, 1994, 1999). « En 1914, les 300000 tonnes de bauxite extraites en Provence représentent les trois-quarts de la production mondiale $»^{20}$. Ils notent aussi que ce patrimoine a été effacé matériellement de la ville et symboliquement des mémoires. Ce phénomène d'effacement et de cryptomnésie est intéressant à questionner et à mettre en relation avec la question de la fragmentation des territoires et des mémoires : réactualisation et mise en lumière à Gardanne, oubli durable à Marseille reconvertie en « ville culturelle $»^{21}$.

Le thème des déchets industriels illustre la complexité des tressages entre territoires réels et imaginaires. Les territoires s’imbriquent les uns dans les autres : le territoire industriel arrimé au principe de réalité économique : la production, les emplois, l'ingénierie et l'innovation industrielles, etc., le territoire-mer au principe de réalité des activités traditionnelles : la pêche ${ }^{22}$, le territoire des espaces protégés, sanctuaires «verts et bleus» mais aussi réponse «opérationnalisée» à l'émergence d'une culture de l'écoresponsabilité. La mer-territoire de la Méditerranée est une mosaïque de domaines d'activités qui sont liés à des imaginaires thématisés, fragmentés. La mer du quotidien (Roux 1997), celle des travailleurs de la mer (pêcheurs, marins, etc.) des habitants du littoral se distingue d'autres représentations de la mer, celles de la mer - loisir (tourisme, plaisance et « rivages enchanteurs »), celles qui ont à voir avec les grands intérêts économiques, géostratégiques, militaires. La mer est un linceul des déchets pour tous, les villes et métropoles du littoral, les industriels (dont l'industrie du tourisme), les transports maritimes. Les problématiques de l'halieutique et des activités liées à la mer, mais aussi d'une certaine banalisation des mers comme mer-territoires "balisés " et stratégiques, conduisent les géographes (cf. notamment : Trouillet 2006) à appeler de leurs vœux la constitution d'une géographie humaine et sociale des mers. Les chercheurs par leurs travaux deviennent des «parties prenantes » à part entière et contribuent à montrer, à mesurer souvent ce qui reste pour la grande majorité des humains invisible (écotoxicologie, écotoxicité aquatique, biodiversité, écologie marine, etc.).

Nous venons de voir combien cet objet encombrant matériellement et symboliquement,ledéchet,estexemplairedestensionsentreterritoiresetintérêts économiques (production, consommation) vs enjeux environnementaux. Les

20 Collectif : Rives nord-méditerranéennes, 2000.

21 Cf. n̊114 (2010) de la revue Méditerranée.

22 En réalité, deux grands modèles de pêche cohabitent en Méditerranée comme ailleurs sur les océans : la pêche traditionnelle, la pêche industrielle. 
territoires de l'activité économique sont à la fois circonscrits (on peut localiser des sites d'activités et de production - distribution) et sans frontières, dans le sens où les flux de toute nature circulent dans des espaces a-territorialisés. Les déchets des activités industrielles et de services, de la consommation, leurs rejets, la circulation de ces rejets, l'abandon, l'enfouissement, le traitement, le recyclage sont autant de thèmes qui soulignent combien le déchet industriel est au bout du compte emblématique de l'interpénétration des territoires matériels et symboliques, économiques, environnementaux, mais aussi culturels et sociétaux.

\section{Alerter, expérimenter, innover, historiciser, symboliser: formes inter-organisationnelles et "territoires de compétences»}

Bien souvent les acteurs de l'alerte, de l'information et de la sensibilisation sont des chercheurs, des pouvoirs publics, des membres de la société civile et certains médias. Nous allons compléter l'illustration de ce point avec un bref rappel d'une autre thématique préoccupante concernant les déchets en mer, le déchet plastique.

Des contributions et informations scientifiques, publiques et médiatiques s'accumulent témoignant de la dégradation toujours plus avancée des eaux de la Méditerranée et de ses conséquences sur la chaîne alimentaire et biologique. La lutte contre le déchet plastique, rejeté en mer depuis 50 ans et désormais présent dans toutes les mers et tous les océans du globe, est devenue une cause largement publicisée 23 avec l'annonce $d u$ " $7^{\mathrm{e}}$ continent » dans le Pacifique (3,5 millions de $\mathrm{km}^{2}$, entre la Californie et Hawai). En ce qui concerne la Méditerranée, parmi les contributions publiques, le rapport Courteau, du nom du sénateur qui l'a rédigé24, par son ampleur et sa rigueur marque une étape importante - audition de deux cents scientifiques français et étrangers, visite de plusieurs pays riverains du bassin (Grèce,Turquie, Malte, Tunisie, Libye...) -. L'auteur précise que : " plus de $80 \%$ de la pollution maritime en Méditerranée provient des terres » et que, si rien n'est fait, 2030 marquera un tournant irréversible. Le déchet plastique à lui tout seul témoigne de la globalisation des échanges et donc des échanges de déchets partout sur les océans. On peut, en ce qui concerne la Méditerranée, souligner que la question des échanges dès l'Antiquité, a été explorée par les historiens (histoire économique) autour de la traçabilité d'un objet-déchet, moins polluant mais très présent, l'amphore. Ce bref rappel souligne une fois de plus que la problématique des déchets n'est pas toute récente (cf. également : Béguin, 2013), même si les déchets, avec les révolutions industrielles et les vagues de

$23 \mathrm{http}: / /$ fresques.ina.fr/jalons/fiche-media/InaEdu05232/dechets-plastiques-dans-le-pacifique-un-7econtinent.html ; Dupont G., 2010, Un « continent » de déchets plastiques a été découvert dans l'Atlantique nord [archive], Le Monde, 5 mars.

24 Cf. : La pollution de la Méditerranée : état et perspectives à l'horizon 2030, résumé du rapport de M. Roland Courteau, Sénateur de l'Aude http://www.senat.fr/rap/r10-652/r10-652-syn.pdf. 
mondialisation des échanges, ont radicalement changé de nature, de toxicité et de volume.

Les contributions centrées sur l'alerte, l'information, la sensibilisation sont donc nombreuses. Mais, comme bien souvent, l'action semble en retrait des déclarations publiques et privées et des emballements médiatiques. Au bout du compte, nous pouvons nous demander quelles sont les relations entre la sphère de l'information et des connaissances et la sphère des décisions, des actions (et des financements de ces actions).

Dès lors que l'action est en cause, il apparaît que celle-ci prend forme plus aisément lorsque d'autres acteurs entrent en scène. Le thème des déchets que nous avons choisi montre à lui tout seul combien les enjeux environnementaux sont liés à l'émergence et à la coordination de formes que nous qualifions d'inter-organisationnelles. Ces formes sont composées d'entreprises, de collectivités locales - de pouvoirs publics, de professionnels des déchets, de riverains, d'associations de protection de l'environnement, de médias, de chercheurs, etc. dès lors qu'ils coopèrent. Il s'agit bien d'initier des formes élargies favorisant des échanges, des connexions entre des ressources, cognitives, matérielles (techniques, financières), humaines et symboliques.

Cependant, ces connexions ne se font pas «naturellement » ni spontanément. Des obstacles culturels (cultures organisationnelles et de métiers, valeurs et éthique ${ }^{25}$ ) et téléologiques (divergence des objectifs, des temporalités) existent (cf. Bernard \& Caspar 2010). Certains dispositifs jouent des rôles de facilitateurs (appels à projets, mise en place de structures à vocation fédératrices notamment), ils sont plutôt portés par les pouvoirs publics à différentes échelles territoriales (régionales, nationales, internationales...). Les instances internationales se mobilisent, le Programme des Nations Unies pour l'Environnement (PNUE) propose un plan de gestion des déchets solides en Méditerranée. L'Union pour la Méditerranée, liant les pays de la Méditerranée et les États membres de l'Union européenne (43 pays et 775 millions d'habitants) avait connu un lancement fortement médiatisé au Sommet de Paris pour la Méditerranée en 2008 et affichait une ambition économique large, intégrant le DD, depuis les effets se sont dissipés.

Des initiatives sont prises également par la société civile et donc aussi par des acteurs économiques ${ }^{26}$, initiatives qui sont encore difficiles à cartographier de manière exhaustive, à catégoriser et à évaluer précisément.

Sur le terrain économique, les réseaux techno-industriels de l'ingénierie "verte » se constituent peu à peu rassemblant des " parties prenantes » de l'innovation industrielle. Quelques entreprises commencent à se spécialiser pour récupérer les déchets plastiques en mer et les recycler, mais la visibilité

25 Cf. Gardère E. \& Gramaccia G. (2005).

26 Une mention particulière pour les initiatives du groupe Ricard, avec ce qui s'appelle désormais « l'Institut océanique Ricard /.../qui a conquis ses lettres de noblesse dans le monde de la recherche internationale " (Domenicho, 2006). 
scientifique et médiatique n'est pas très bonne quant à la conduite de ces activités. Nous pouvons aussi prendre l'exemple du « Pôle Mer Méditerranée » partenaire de la structuration de la filière du " génie écologique côtier » qui rassemble à la fois des grands groupes comme Véolia, Suez Environnement, EGIS et de nombreuses PME et laboratoires de recherche qui cherchent ensemble à conjuguer spécificités économique et technologique des « territoires côtiers » et responsabilité environnementale. Des PME sont concernées par d'autres projets, en 2014, les Échos présentent certains de ces projets « de réhabilitation de sites marins dégradés /.../. Le marché concerne des centaines d'ouvrages rien qu'en Méditerranée $»^{27}$.

D'autres expérimentations à orientation scientifique et sociétale, modestes mais au rayon d'action international et au potentiel symbolique marqué, sont conduites. Parmi celles-ci, on note la relative médiatisation de l'expédition scientifique Tara (de mai à novembre 2014) qui «/confirme/ une pollution plastique inquiétante en Méditerranée ${ }^{28}$. Cette expédition, conçue à la fois comme une expédition scientifique et un dispositif de sensibilisation, avait fait escale dans 13 pays du pourtour méditerranéen, à cette occasion, la goélette avait pu être visitée par environ 10000 personnes dont des scolaires.

Des réseaux associatifs de l'éducation et de la sensibilisation àl'environnement du côté de la société civile ont capitalisé une expérience intéressante. Nous pouvons donner l'exemple d'Eco gestes Méditerranée. Cette association rassemble « 25 structures d'éducation à l'environnement mettent en commun leurs compétences et leurs moyens pour intervenir sur les côtes de PACA, Corse et Languedoc-Roussillon ». La mission est ainsi définie : " expliquer, promouvoir et diffuser les gestes pratiques pour préserver la Méditerranée ». Les associations peuvent être étroitement liées à des observatoires locaux, par exemple pour Ecogestes Méditerranée, l'Observatoire marin de la communauté de communes du Golfe de Saint Tropez $z^{29}$.

Ces types de réseau -entrepreunarial, associatif- ont en commun de coopérer régulièrement avec des chercheurs. Cependant ils sont peu connectés entre eux, malgré des tentatives de mise en relation. À cette étape, la question que nous posons est la suivante: $\mathrm{y}$ a-t-il d'autres expérimentations qui permettent de franchir les frontières entre réseaux?

À nouveau, nous essayons d'identifier des cas concrets, que nous regroupons en « territoires éducatifs » et en « territoires de compétences » qui montrent le rôle de mise en relation, d'inter-médiation effectué par les pouvoirs publics.

Du côté des territoires éducatifs et symboliques, le Réseau $\mathrm{Mer}^{30}$ avait été créé par le Conseil de Région PACA au début des années 2000. « Ce réseau

27 http://www.lesechos.fr/26/05/2014/lesechos.fr/0203523703407_le-genie-ecologique-marin-s-invite-dansles-chantiers-cotiers-mediterraneens.htm\#C5Qw5pituw6wmqKJ.99

$28 \mathrm{http}: / /$ www.20minutes.fr/planete/1486831-20141124-tara-revele-pollution-plastique-inquietantemediterranee

29 http://www.observatoire-marin.com/om_historique.htm

$30 \mathrm{http} / / /$ www.reseaumer.org/ 
rassemble différents types d'acteurs: des associations et des structures de gestion de la zone côtière qui font de l'éducation à l'environnement marin et littoral, mais aussi des représentants des institutions (services de l'État et collectivités locales), de la communauté scientifique, des professionnels des sports nautiques et du tourisme ». Des dispositifs de mise en relation sont mis en place, par exemple celui de la charte qui co-définit les missions et les valeurs du réseau. Parallèlement, des ONG conduisent des opérations concrètes et à visée éducative et à portée symbolique, par exemple consacrées $\mathrm{au}$ « nettoyage de la Méditerranée » ${ }^{31}$.

Par ailleurs, la notion de « territoires de compétences ${ }^{32}$ semble intéressante pour aider à passer certaines frontières entre professions, organisations, structures publiques - privées, etc. Un dispositif empirique représentatif de cette démarche est constitué par Le Technopôle de l'environnement ArboisMéditerranée, créé en 1991 à l'initiative du Conseil Général des Bouchesdu-Rhône. Ce Technopôle définit ses ressources de la manière suivante : «Le Technopôle accueille 900 salariés, 400 chercheurs et 300 étudiants (Master et doctorants)/.../ 110 entreprises, 12 laboratoires de recherche/.../ 8 pôles de compétitivité/.../ ». Le Technopôle a vocation à développer les liens entre la recherche académique et la recherche privée. En 2010, parmi les publications du laboratoire ECCOREV, membre du Technopôle, on note la formulation d'un nouveau domaine de la géographie, celui d'une " géographie des interfaces " (Lampin-Maillet et alii, 2010).

Ce qui peut être retenu de ces quelques exemples, c'est qu'ils ont en commun d'expérimenter des coopérations, des connexions entre organisations aux cultures et compétences différentes. Ce point est essentiel pour comprendre comment l'action peut se développer dans des projets associant savoirs et terrains, prenant en compte systématiquement les thématiques environnementales pour préserver les formes de vie, et donc d'activités, qui nécessitent de penser les interdépendances multiples entre «territoires côtiers» et «mer- territoires».

\section{Conclusion}

«La Nature est un temple /.../ L'homme y passe à travers des forêts de symboles" (Baudelaire, 1857) ${ }^{33}$.

Penser dans les catégories de "Méditerranée » suppose de prendre en compte l'altérité. La " mer au milieu des terres » invite à un exercice continu de mise en relation diachronique et synchronique (Bernard \& Durampart 2013). Penser la relation nature - culture et donc la présence de l'humain

31 «L'ONG Sea Sheperd a lancé l'opération Mare Nostrum, 13 juillet 2015 à Marseille. Elle a pour but de nettoyer la Méditerranée de ses incalculables déchets ».

32 Notion que l'on retrouve dans la loi portant la nouvelle organisation territoriale.

33 Charles Baudelaire, Correspondances, Les Fleurs du mal,1857. 
dans le monde conduit à comprendre les dispositifs, les organisations et les institutions produites par les humains pour mener leurs activités et assurer le maintien et le développement de leurs sociétés.

Pour ce thème environnemental, comme pour bien d'autres, nous retrouvons les bornes de l'agir et du symboliser. L'action est capitale, elle sera d'autant plus durable qu'elle sera rapportée à un niveau d'identification élevée (Wegner \& Vallacher 1986) où elle prend un sens susceptible de transcender les limites de l'acte isolé, technique et thématisé. Les processus de symbolisation ${ }^{34}$ sont donc essentiels pour inscrire l'action dans la durée, mais surtout pour mettre en relation et en vibration les cours d'action avec les chaînes de significations sans lesquelles l'acte pro environnemental perd son sens. Ainsi, agir pour la préservation et la restauration des environnements naturels méditerranéens, c'est s'inscrire inévitablement dans une perspective symbolique au sens où Daniel Bougnoux le proposait: «Le monde symbolique est le monde de la culture que nous ajoutons à la nature »(Bougnoux 2008: 16). La dimension symbolique pour les questions environnementales en Méditerranée joue avec les représentations des territoires locaux (terroirs) - globalisés (ensemble des pays riverains ; espace marin). De ce point de vue, l'objet du déchet marin par sa circulation trans-territoires, mais aussi parce qu'il est pris dans un réseau dense de circulation socio sémiotique et médiatique (symbole des excès - et des inégalités - dans la production et la consommation de biens) est un objet d'études qui semble bien relever de ce que Braudel appelait «la civilisation matérielle».

\section{Corpus numérique (extrait) :}

\section{À propos des déchets:}

http://www.seashepherd.fr/

http://www.expeditionmed.eu/fr/

http://www.notre-planete.info/actualites/4139-dechets-plastiquesmediterranee

http://www.midilibre.fr/2016/01/03/dechets-deverses-en-mediterranee

http://planbleu.org/

http://planbleu.org/fr/medcop21-le-forum-de-la-societe-civilemediterraneenne-pour-le-climat

http://fresques.ina.fr/jalons/fiche-media/InaEdu05232/dechetsplastiques-dans-le-pacifique-un-7e-continent.html

\section{Boues rouges - Corse :}

http://m3c.univ-corse.fr/omeka/medias-et-mobilisations-en-corse-de1945-a-nos-jours 


\section{Fresques:}

http://sites.ina.fr/mobilisations-en-corse/

\section{Tempo:}

http://sites.ina.fr/mobilisations-en-corse/tempo/

\section{Réseaux scientifiques et parties prenantes:}

http://www.arbois-med.com/

https://www.youtube.com/playlist?list=PLIjqlgLGPIILZ3rgX60ocnTxVg J8gkUE8

http://mediamed.mmsh.univ-aix.fr/chaines/ramses2/penser-laMediterranee

www.maxisciences.com/méditerranée/mediterranee-un-plan-pourpreserver-sa-biodiversite_art $9463 . h t m l$

http://www.institut-paul-ricard.org/?Dechets-en-Mediterranee

www.conservatoire-du-littoral.fr/40-les-delegations.htm

\section{BIBLIOGRAPHIE}

BABY-COLLIN V., BENSAÂD A. \& SINTÈS P. (coord.), Migrations et territoires de la mobilité en Méditerranée, Méditerranée, Revue géographique des pays méditerranéens, $\mathrm{n}^{\circ} 113,2009$.

BATAZZI C. \& MASONI LACROIX C. (sous la dir.), «Communication, organisation, symboles», MEI, n 29, Paris, L'harmattan, 2008.

BÉGUIN M., "L'histoire des ordures : de la préhistoire à la fin du dix-neuvième siècle ", VertigO - la revue électronique en sciences de l'environnement [En ligne], Volume 13 Numéro 3 | décembre 2013, mis en ligne le 30 décembre 2013. URL : http://vertigo.revues.org/14419; DOI : 10.4000/vertigo.14419

BERNARD F. \& DURAMPART M. (sous la dir.), Savoirs en action. Culture et réseaux méditerranéens, Paris, CNRS Éditions, 2013.

BERNARD F., Environnement-communication- organisations- institutions: Quels apports et approches critiques? In Thomas Heller, Romain Huet, Bénédicte Vidaillet, Communications-organisations et pensées critiques, Villeneuve d'Ascq, Presses universitaires du Septentrion, 2013, p. 433- 444.

BERNARD F. \& CASPAR M., « La recherche-action participative et coopérative. Eléments d'expérimentations, de bilans et perspectives ", Faire savoirs, 9, 2010, p. 45-54.

BOUGNOUX D., "Nous ne sommes pas inconditionnellement des animaux symboliques ». Entretien avec Claudine Batazzi \& Céline Masoni Lacroix, in C. Batazzi \& C.Masoni Lacroix, Communication, organisation, symboles. MEI, n’ 29, Paris, L'harmattan, 2008, p.7-17.

BRAUDEL F., Écrits sur l'Histoire, Paris, Flammarion, 1977.

BRAUDEL F., « Histoire et Sciences sociales. La longue durée », Annales ESC, vol. 4, 1958, p.725-753. 
CASTORIADIS C., L'institution imaginaire de la société, Paris, Seuil, 1975.

COURTEAU R., La pollution de la Méditerranée: état et perspectives à l'horizon 2030, Office parlementaire d'évaluation des choix scientifiques et technologiques, $n^{\circ} 652$ (20102011) - 21 juin 2011 ; en version courte et en ligne : http://www.senat.fr/rap/r10-652/ r10-652-syn.pdf

DI MÉO G., Géographie sociale et Territoires, Paris, Nathan Université, 1998.

DOMENICHINO J., « Notice biographique de Paul Ricard », Méditerranée [Online], 106 | 2006, Online since 16 October 2008. URL : http://mediterranee.revues.org/416

D'ORAZIO L., «Une île de Violence: le traitement médiatique du "Problème corse” (1965-2007)», Rives méditerranéennes [En ligne], Jeunes chercheurs, mis en ligne le 15 juin 2011. URL : http://rives.revues.org/3956

FABRE T. (sous la dir.), Penser la Méditerranée au XXI ${ }^{\mathrm{e}}$ siècle, $20^{\mathrm{e}}$ Rencontres d'Averroès, Collection: Rencontres d'Averroès, 2014.

GARDÈRE E. \& GRAMACCIA G., « La communication des nouvelles éthiques de l'entreprise », Communication E Organisation [En ligne], 26 | 2005, mis en ligne le 19 juin 2012. URL : http://communicationorganisation.revues.org/3267

GRÉSILLON B. (coord.), «Villes culturelles en Méditerranée », Méditerranée, Revue géographique des pays méditerranéens, $\mathrm{n}^{\circ} 114,2010$.

LAMPIN-MAILLET C., PÉREZ S., FERRIER J.-P., ALLARD P., Géographie des interfaces : une nouvelle vision des territoires, Paris, Éditions Quae, 2010.

LARDELLIER P., Formes en devenir, approches technologiques, communicationnelles et symbolique. Paris, ISTE Éditions, 2014.

MCCOMBS M. \& SHAW D., "The agenda-setting function of mass media », Public Opinion Quarterly, vol. 36, n 2, 1972, p. 176-187.

MENDEZ A. \& MERCIER D., "Compétences-clés de territoires. Le rôle des relations interorganisationnelles», Revue française de gestion 5/2006 ( $\left.\mathrm{n}^{\circ} 164\right)$, p. 253275 URL : www.cairn.info/revue-francaise-de-gestion-2006-5-page-253.htm.

MORIN E., «Penser la Méditerranée et méditerranéiser la pensée», CONFLUENCES Méditerranée - n²8, hiver 1998-1999, p. 33-47.

ROUX M., L'Imaginaire marin des Français. Mythe et géographie de la mer, Paris, L'Harmattan, 1997.

HOPKINS A. G. (edited by), Globalization in World History, Londres, PimliCo, 2002.

STORY J., Le système mondial de Susan Strange, Politique étrangère, 2/2001, p.433-447.

TROUILLET B., «Réglementation de l'exercice de la pêche et dynamiques d'occupation de la mer côtière», in CHAUSSADE J. \& GUILLAUME J. (coord.), Pêche et aquaculture. Pour une exploitation durable des ressources vivantes de la mer et du littoral, actes du colloque international, Géolittomer (LETG-UMR 6554, CNRS), Nantes, janvier 2004, Rennes, PUR, 2006.

WEGNER D. M.\&VALLACHER R.R.,Action identification, in R.M. Sorrentino., \& E.T. Higgins (Eds.), Handbook of motivation and cognition: foundations of social behavior, New York, Guilford, 1986, p. 550-582. 
Résumé : L'auteure montre les interrelations entre les notions d'écoresponsabilité, de territoires, de cultures et de formes interorganisationnelles. La réflexion porte sur les enjeux et problématiques associés de l'action et de la symbolisation. Elle s'inscrit dans des travaux consacrés aux espaces marins et littoraux méditerranéens. Le cas des déchets est plus particulièrement étudié.

Mots-clés: Méditerranée, déchets, médiatisations, inter-territoires, formes interorganisationnelles.

Abstract : The author shows the interrelations between the notions of ecoresponsibility, territories, cultures and interorganizational forms. The analysis concerns the stakes and the problems associated of the action and the symbolization. It joins in works dedicated to the Mediterranean marine and littoral spaces. The case of waste is more particularly studied.

Keywords: Mediterranean Sea, wastes, mediatizations, inter-territories, interorganizational forms. 
